The Astrophysical Journal, 181: 51-59, 1973 April 1

(C) 1973. The American Astronomical Society. All rights reserved. Printed in U.S.A.

\title{
GAS MOTIONS IN THE NUCLEUS OF THE SEYFERT GALAXY NGC 4151
}

\section{MARIE-HELENE UlRICH}

University of Texas at Austin, and Département d'Astrophysique Fondamentale de Meudon Received 1972 June 9; revised 1972 November 2

\section{ABSTRACT}

Image-tube spectra with a dispersion of $28 \AA \mathrm{mm}^{-1}$ and a scale of 13 ". $5 \mathrm{~mm}^{-1}$ in the direction perpendicular to the dispersion, have been taken in 11 different position angles across the nucleus of NGC 4151. The structure of the [O III] lines $\lambda \lambda 4959,5007$ varies with position angle; to simplify the description of the line structure we have distinguished four components corresponding to four main clouds (I-IV) with different dimensions, locations, and velocities with respect to the center of the galaxy. About 50 percent of the energy emitted in the [O III] lines comes from cloud I which is exactly centered on the region emitting the continuum. Cloud II, the brightest after cloud I, is centered at $\sim 1^{\prime \prime} .5$ from the continuum source in P.A. $240^{\circ}$; the two weaker clouds III and IV are located in the NE quadrant. Clouds II, III, and IV are roughly aligned along the minor axis of the galaxy; therefore, the motions indicated by the spectra are radial rather than rotational. If these motions are interpreted as expansion motions from the center, cloud II is moving away from the center and toward us with an apparent velocity of $180 \mathrm{~km} \mathrm{~s}^{-1}$ and clouds III and IV are moving in the opposite direction to cloud II with respect to the center of the galaxy.

The four brightest clouds of ionized gas extend over less than 4" (200 pc) from the center of the galaxy, i.e. over smaller distances than in NGC 1068. Also, their velocities with respect to the center and their expansion velocities are in general of the order of $200-300 \mathrm{~km} \mathrm{~s}^{-1}$; again, smaller than in NGC 1068.

Subject headings: galactic nuclei - galaxies, motions in - Seyfert galaxies

\section{INTRODUCTION AND OBSERVATIONS}

In the last few years several observations have shown that expansion motions occur in the central regions of some galaxies (see review paper by Burbidge 1970). In this respect, investigation of nearby galaxies is of particular interest because the velocity fields in their nuclei can be observed with the best spatial resolution.

This is a report of some spectrographic observations of the nucleus of the Seyfert galaxy NGC 4151 and a qualitative description of the properties of the nuclear clouds of ionized gas as can be deduced from the structure of the emission lines. The observing procedure is described below. The geometry and the velocity field in the cloud complex are analyzed in $\S$ II. The results are discussed in $\S$ III.

Walker (1968a) observed the nucleus of NGC 4151 at the coude focus of the 120inch $(300-\mathrm{cm})$ telescope at Lick Observatory with the Lallemand electronographic camera. Walker found that besides a gas cloud centered on the region emitting the continuum, another cloud was present SW of the center of the galaxy and had a velocity of $235 \mathrm{~km} \mathrm{~s}^{-1}$ with respect to the central cloud. The nucleus of NGC 4151 has been reobserved with a better scale than was available to Walker, and with deeper exposures in an attempt to see whether other fainter gas clouds are also present. The spectra have been taken in various position angles with the nucleus held stationary on the slit. They have been obtained with the Meinel image-tube spectrograph attached to the $\mathrm{f} / 18$ Cassegrain focus of the $107-$ inch $(273-\mathrm{cm})$ telescope at McDonald Observatory. On the spectrograms the dispersion is $28 \AA \mathrm{mm}^{-1}$ and the scale perpendicular to the dispersion is $13.1 \mathrm{~mm}^{-1}$. The resolution element of the image-tube spectra is 
approximately $40 \mu$, corresponding to a resolution of 0 ".55 on the sky. The slit width was set at $120 \mu$ or $150 \mu$ depending on the night. With a slit width of $150 \mu$, the full width of a rather strong comparison line measured on the plate is $65 \mu$, equivalent to $1.8 \AA$ with the dispersion used. During the observations, the diameter of the seeingdisk was between 1" and 1.5". Observational data are listed in table 1. Several spectra were recorded on each plate, and are distinguished from others on that plate by alphabetic lettering ( $a, b$, c, etc.). Most of the spectra were taken on baked Kodak IIaO emulsion. The spectra on plate 894 were obtained on unbaked emulsion and through cirrus, and as a result their photographic density is similar to that of the 12-minute exposure spectra.

Figure 1 (plate 4) shows the range $\mathrm{H} \beta-[\mathrm{O}$ III] $\lambda \lambda 4959,5007$ of the spectra 894a,b,c. Figures $2 a$ and $2 b$ (plates 5 and 6 ) show very high enlargements of the region of the [O III] lines of the 5- and 25-minute spectra taken on 1972 February 18, except that spectrum 861a which was not correctly guided has been replaced by $862 \mathrm{~d}$. On these figures, the numbers on the left-hand side give the position angle of the top of each spectrum, and the full length of the vertical line on the upper right corner represents $10^{\prime \prime}$.

The aspect of the [O III] lines varies with the position angle of the spectra, complex structure being present on spectra crossing the NE and SW quadrants. In order to describe the line structure in a simple way, four components have been distinguished in the [O III] lines corresponding to four gas clouds.

\section{THE GAS CLOUDS IN THE NUCLEUS OF NGC 4151}

\section{a) Structure of the Emission Lines}

The continuum visible on the spectra comes from a region which is seeing-limited in size. It is likely that this region coincides with the region photographed by Stratoscope

TABLE 1

Spectrographic ObServations of the Nucleus of NGC 4151

\begin{tabular}{|c|c|c|c|c|c|}
\hline Spectrum & Date (UT) & $\begin{array}{l}\text { Position Angle } \\
\text { (degree) }\end{array}$ & $\begin{array}{l}\text { Exposure } \\
\text { (minutes) }\end{array}$ & Spectral Range & $\begin{array}{l}\text { Slit Width } \\
\text { (microns) }\end{array}$ \\
\hline 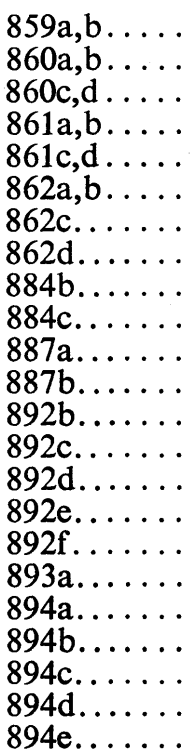 & $\begin{array}{l}1972 \text { Mar. } 16 \\
1972 \text { Mar. } 17 \\
1972 \text { Mar. } 19\end{array}$ & $\begin{array}{r}0 \\
30 \\
60 \\
90 \\
120 \\
150 \\
180 \\
90 \\
270 \\
0 \\
90 \\
0 \\
240 \\
270 \\
300 \\
330 \\
0 \\
240 \\
75 \\
45 \\
25 \\
355 \\
325\end{array}$ & $\begin{array}{c}5 \text { and } 25 \\
5 \text { and } 25 \\
5 \text { and } 25 \\
5 \text { and } 25 \\
5 \text { and } 25 \\
5 \text { and } 25 \\
12 \\
12 \\
60 \\
60 \\
10 \\
10 \\
10 \\
10 \\
10 \\
10 \\
10 \\
10 \\
25 \text { (cirrus) } \\
25 \text { (cirrus) } \\
25 \text { (cirrus) } \\
25 \text { (cirrus) } \\
25 \text { (cirrus) }\end{array}$ & 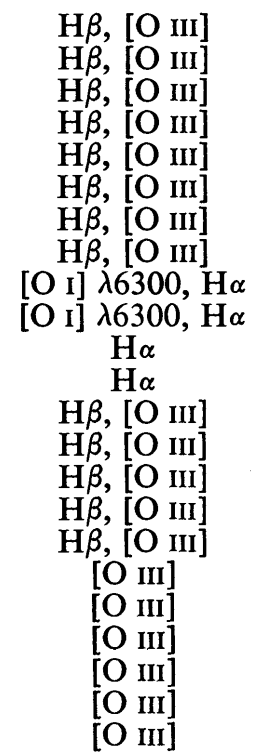 & $\begin{array}{l}150 \\
150 \\
150 \\
150 \\
150 \\
150 \\
150 \\
150 \\
150 \\
150 \\
120 \\
120 \\
150 \\
150 \\
150 \\
150 \\
150 \\
150 \\
120 \\
120 \\
120 \\
120 \\
120\end{array}$ \\
\hline
\end{tabular}


II and for which a conservative upper limit of 0 ".08 is given for the half-power width (Schwarzschild 1971). In the following, the source of continuum is referred to as the center of the galaxy and is taken as the origin of the distances measured in the direction perpendicular to the dispersion.

On very short exposure spectra, the images of the [O III] lines $\lambda \lambda 4959,5007$ appear exactly on the continuum. With increasing exposure time, this component-or component I-becomes denser and larger but remains symmetrical with respect to the continuum regardless of position angle; thus, it originates in a cloud-cloud Iwhich, in projection on the sky, is centered on the region emitting the continuum.

Component II is blueshifted with respect to component I and appears in position angles in the range $205^{\circ}-270^{\circ}$ and reaches its maximum radial extent in P.A. $240^{\circ}$ (see figs. $2 a$ and $2 b$ ). Cloud II, which corresponds to this second component, partly overlaps cloud I; in particular, in P.A. $270^{\circ}$ and $300^{\circ}$, only the difference in wavelength makes component II distinct from the central component I. This is in excellent agreement with Walker's results.

In the NE quadrant there is no counterpart exactly symmetrical to cloud II with respect to the center. Two weak features are visible in the NE quadrant; they are best seen on spectra taken in P.A. $25^{\circ}-45^{\circ}$ (see fig. 1). One is a narrow component redshifted by $\sim 2.6 \AA$ with respect to the central wavelength of component I and which extends over $\sim 3^{\prime \prime}$ in the range of position angles $25^{\circ}-60^{\circ}$. The corresponding cloud, cloud III, is fainter and more extended than cloud II. The other feature present in the NE quadrant also appears redward of component I; it is a relatively broad feature extending over $8 \AA$ and is not centered on the continuum. It corresponds to cloud IV which is seeing-limited in size and is centered $\sim 1^{\prime \prime} \mathrm{NE}$ of the center of the galaxy.

The four components which we have distinguished in the complex structure of the [O III] lines are schematically represented in figure 3. The sketch is valid for the spectra taken in P.A. $\simeq 45^{\circ}$.

No useful information has been obtained from the other emission lines: $\mathrm{H} \beta$ and [O I] $\lambda 6300$ are too faint on our spectra. Some structure is present in $\mathrm{H} \alpha$ but is made indistinct by the intense wings.

\section{b) Measures of the Wavelengths and Radial Extents}

The measures have been made with a conventional one-dimensional measuring engine. The wavelengths and the radial extents were obtained in two sets of measurements made with the plates oriented in two perpendicular directions. No correction for distortion has been applied since the distances involved in measuring the radial extents of the components and the relative velocities of the various clouds are very small, not exceeding $1 \mathrm{~mm}$. Also, the images of the [O III] lines on spectra taken on different nights had been formed on different areas of the photocathode, and from plate to plate taken in the same position angle, there is no systematic difference in the relative locations of the various components.

The radial extents of the components in various position angles were measured on the spectra which have the best spatial resolution, and are listed in table 2 .

The wavelengths of six features of the [O III] lines have been measured on the spectra which have the best wavelength resolution. The wavelength shifts of the six features, i.e. the differences between the measured wavelengths and the rest wavelength of the [O III] lines, are listed in table 3, after correction for the earth's motion around the Sun.

The central wavelengths of components I and III are defined without ambiguity because the components are nearly symmetrical. The measures at the blue edge of component II, blue and red edges of component I, and red edge of component IV have been made specifically at the location of a strong gradient of photographic 


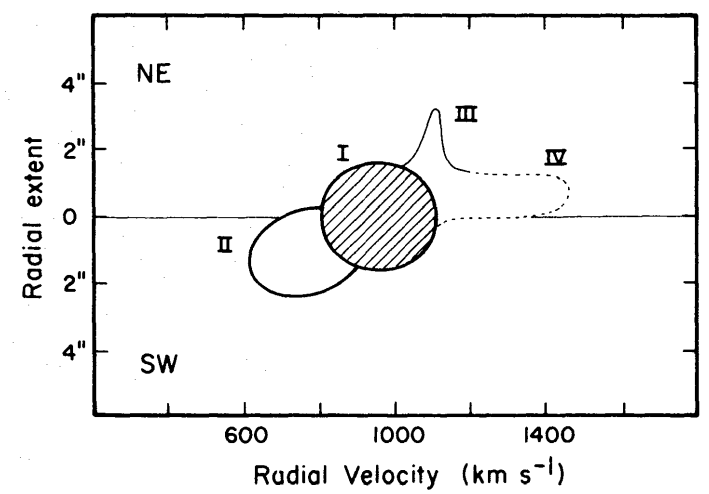

FIG. 3.- Schematic representation of the line [O III] $\lambda 5007$ emitted by the nucleus NGC 4151, in P.A. $45^{\circ}$ (see fig. 1). The four components which we have distinguished in the line are identified by the numbers I to IV. Hatched area, component I; thick continuous line, component II; thin continuous line, component III; thin broken line, component IV. These notations are used in fig. 4, below, to identify the four clouds from which the components originate. The thin horizontal line represents the middle of the continuum and is the origin of the radial extent. The numerical values of the radial extents (ordinates) and of the radial velocities reduced to the Sun (abscissae) are taken from tables 2 and 3 , respectively.

density. These measures have been made in attempt to determine the velocity dispersion in clouds II, I, and IV. This is made difficult by three factors. First, it is likely that the velocity distribution in the clouds has no sharp cutoff. Second, the atmospheric seeing causes some inevitable blurring of the various components. The third difficulty is inherent in the use of the photographic emulsion on which the image size of a line depends not only on the intrinsic line-width but also on the exposure time and the line intensity. Similar difficulties prevent an accurate determination of the radial extent of the various clouds. Nevertheless, the scatter of the data of tables 2 and 3 is relatively small, implying that the features measured correspond to physical characteristics of the clouds.

TABLE 2

Radial EXTENT OF THE COMPONENTS OF THE [O III] LiNES (in seconds of arc)

\begin{tabular}{|c|c|c|c|c|}
\hline $\begin{array}{l}\text { Position Angle } \\
\text { (degrees) }\end{array}$ & $\underset{\text { I }}{\text { Component }}$ & $\begin{array}{l}\text { Component } \\
\text { II }\end{array}$ & $\begin{array}{l}\text { Component } \\
\text { III }\end{array}$ & $\begin{array}{l}\text { Component } \\
\text { IV }\end{array}$ \\
\hline 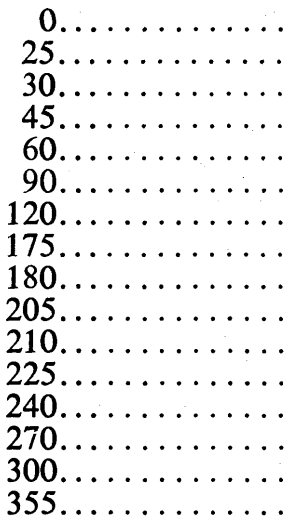 & $\begin{array}{l}2 \\
1.0^{*} \\
2 \\
1.1^{*} \\
1.5^{*} \\
1.8 \\
2.2 \\
1.3^{*} \\
2 \\
1.3^{*} \\
1.1^{*} \\
1.5 \\
2.2 \\
2.2 \\
1.4^{*}\end{array}$ & $\begin{array}{l}\cdots \\
\cdots \\
\cdots \\
\cdots \\
\cdots \\
\cdots \\
\cdots \\
2 * \\
2.5 \\
2.5^{*} \\
2.7 \\
1.8 \\
\cdots \\
\cdots\end{array}$ & $\begin{array}{l}3.3 \\
3.0^{*} \\
3.5 \\
2.8^{*} \\
3.5 \\
2.8 \\
\ldots \\
\cdots \\
\cdots \\
\cdots \\
\cdots \\
\cdots \\
\ldots \\
\ldots \\
\ldots\end{array}$ & $\begin{array}{l}1.0^{*} \\
1.5 \\
1.0^{*} \\
1.5 \\
\cdots \\
\cdots \\
\cdots \\
\cdots \\
\cdots \\
\cdots \\
\cdots \\
\cdots \\
\cdots \\
\cdots\end{array}$ \\
\hline
\end{tabular}

* From measures of spectra $894 a, b, c, d$. 
TABLE 3

WaVelength-Shifts of the Main Features of the [O III] Lines (in ^̊)

\begin{tabular}{|c|c|c|c|c|c|c|}
\hline \multirow{2}{*}{$\begin{array}{c}\text { SPECTRUM } \\
\text { NUMBER } \\
\text { AND P.A. }\end{array}$} & \multirow{2}{*}{$\begin{array}{l}\text { Cloud II: } \\
\text { Blue EdGE }\end{array}$} & \multicolumn{3}{|c|}{ Cloud I } & \multirow{2}{*}{$\begin{array}{l}\text { Cloud III: } \\
\text { CENTER }\end{array}$} & \multirow{2}{*}{$\begin{array}{l}\text { Cloud IV: } \\
\text { RED EdGE }\end{array}$} \\
\hline & & Blue Edge & Center & Red Edge & & \\
\hline \multirow{2}{*}{$859 a, 0^{\circ} \ldots \ldots$} & $\ldots$ & $14.50 *$ & 16.35 & 18.25 & $\ldots$ & $\cdots$ \\
\hline & 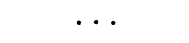 & 14.10 & 16.30 & 17.70 & $\cdots$ & $\cdots$ \\
\hline $859 b, 0^{\circ} \ldots \ldots$ & & 13.75 & 16.45 & 18.50 & $\cdots$ & \\
\hline \multirow{2}{*}{$860 \mathrm{a}, 30^{\circ} \ldots$} & $\begin{array}{c}10.65 \\
\ldots\end{array}$ & 13.80 & $\begin{array}{l}16.35 \\
16.10\end{array}$ & 18.40 & $\cdots$ & 23.35 \\
\hline & $\cdots$ & 14.30 & 16.25 & 18.35 & $\cdots$ & $\cdots$ \\
\hline \multirow{2}{*}{$860 \mathrm{~b}, 30^{\circ} \ldots$} & 9.80 & 13.40 & 16.35 & 18.80 & & \\
\hline & 9.30 & $\cdots$ & & $\cdots$ & 18.80 & 25.50 \\
\hline $860 c, 60^{\circ} \ldots \ldots$ & $\ddot{9} . \dot{80}$ & 14.30 & $\begin{array}{l}16.10 \\
16.50\end{array}$ & 1930 & $\cdots$ & $\cdots$ \\
\hline \multirow{2}{*}{$860 \mathrm{~d}, 60^{\circ} \ldots$} & 10.10 & 13.80 & 16.45 & 19.45 & $\cdots$ & \\
\hline & 8.70 & 12.20 & & $\cdots$ & 18.40 & 25.95 \\
\hline $861 \mathrm{a}, 90^{\circ} \ldots \ldots$ & $\begin{array}{l}\cdots \\
\ldots\end{array}$ & $\begin{array}{l}\ldots \\
\ldots\end{array}$ & $\begin{array}{l}15.75 \\
15.75\end{array}$ & $\cdots$ & $\cdots$ & $\cdots$ \\
\hline \multirow{2}{*}{$861 b, 90^{\circ} \ldots}$. & 10.25 & 13.30 & & 18.70 & $\cdots$ & \\
\hline & $\cdots$ & $\ldots$ & 16.20 & $\ldots$ & $\cdots$ & 24.45 \\
\hline $861 \mathrm{c}, 120^{\circ} \ldots$ & $\begin{array}{l}\cdots \\
\cdots\end{array}$ & $\cdots$ & $\begin{array}{l}15.80 \\
1600\end{array}$ & $\cdots$ & $\cdots$ & $\cdots$ \\
\hline \multirow{2}{*}{$861 \mathrm{~d}, 120^{\circ} \ldots$} & $\ldots$ & $\cdots$ & 15.80 & $\cdots$ & $\cdots$ & $\cdots$ \\
\hline & $\cdots$ & $\cdots$ & 15.95 & $\cdots$ & $\cdots$ & 22.65 \\
\hline \multirow{2}{*}{$862 \mathrm{~b}, 150^{\circ} \ldots$} & $\cdots$ & $\cdots$ & 15.65 & $\cdots$ & $\cdots$ & $\cdots$ \\
\hline & $\cdots$ & $\cdots$ & $\begin{array}{l}16.30 \\
16.25\end{array}$ & $\cdots$ & $\cdots$ & $\cdots$ \\
\hline $862 \mathrm{c}, 180^{\circ} \ldots$ & 9.80 & 12.90 & 16.00 & 19.20 & $\cdots$ & $\cdots$ \\
\hline $862 \mathrm{~d}, 90^{\circ} \ldots$ & $\cdots$ & $\cdots$ & 15.70 & $\cdots$ & $\ldots$ & $\cdots$ \\
\hline \multirow{2}{*}{$892 b, 240^{\circ} \ldots$} & 10.55 & $\begin{array}{l}\cdots \\
\cdots\end{array}$ & $\begin{array}{l}15.75 \\
16.50\end{array}$ & $\begin{array}{l}\cdots \\
\ldots\end{array}$ & $\begin{array}{l}\cdots \\
\ldots\end{array}$ & $\begin{array}{l}\cdots \\
\cdots\end{array}$ \\
\hline & 10.35 & $\ldots$ & 16.50 & $\ldots$ & 19.15 & 24.35 \\
\hline \multirow{2}{*}{$893,240^{\circ} \ldots \ldots$} & 11.75 & & 16.00 & $\ldots$ & & \\
\hline & 10.55 & 15.20 & 625 & $\cdots$ & 18.85 & 24.40 \\
\hline $894 a, 75^{\circ} \ldots \ldots$ & 10.65 & 13.95 & $10.2 J$ & 18.30 & $\begin{array}{l}\cdots \\
\cdots\end{array}$ & $\cdots$ \\
\hline \multirow{2}{*}{$894 \mathrm{~b}, 45^{\circ} \ldots \ldots$} & 1045 & 1420 & 16.10 & $\cdots$ & 1780 & 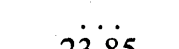 \\
\hline & $10.4 J$ & 14.20 & 16.00 & $\begin{array}{l}\cdots \\
\cdots\end{array}$ & 11.00 & \\
\hline $894 \mathrm{c}, 25^{\circ} \ldots \ldots$ & 10.15 & 14.05 & & $\ldots$ & 18.50 & 25.00 \\
\hline \multirow{2}{*}{$894 d, 355^{\circ} \ldots$} & $\begin{array}{l}\cdots \\
\ldots\end{array}$ & $\cdots$ & 16.35 & $\cdots$ & $\cdots$ & $\cdots$ \\
\hline & $\cdots$ & $\cdots$ & 10.10 & $\cdots$ & $\cdots$ & $\cdots$ \\
\hline
\end{tabular}

Average Wavelength-Shifts (in $\AA$ )

\begin{tabular}{cccccc}
\hline 10.2 & 13.8 & 16.1 & 18.6 & 18.6 & 24.5 \\
$(0.7) \dagger$ & $(0.7)$ & $(0.3)$ & $(0.5)$ & $(0.5)$ & $(1.0)$ \\
\hline
\end{tabular}

Average Radial Velocity Reduced to the Sun (in $\mathrm{km} \mathrm{s}^{-1}$ )

\begin{tabular}{llllll}
\hline 610 & 830 & 970 & 1120 & 1120 & 1470 \\
\hline
\end{tabular}

* Wavelength shift: difference between the measured wavelengths and the rest wavelengths of the [O III] lines, after correction for the Earth's motion around the Sun. Upper line, wavelength shift measured on $\lambda$ 4958.9. Lower line, wavelength shift measured on $\lambda 5006.8$.

$\dagger$ In parentheses: standard deviation of the mean, in $\AA$. 
The wavelength shifts of the features of the [O III] lines listed in table 3 do not vary appreciably with position angle. A mean wavelength shift was obtained by averaging the measures made on $\lambda 4959$ and $\lambda 5007$ and by averaging over the position angle, with the same weight assigned to each individual measure; the mean wavelength shifts are given at the bottom of table 3 together with the standard deviations of the mean in parenthesis. In the last line of table 3 are listed the radial velocities (reduced to the Sun) of the main features of the [O III] lines.

In table 2 the value of the radial extent of a component in a given position angle is the arithmetical mean of the values measured on the two [O III] lines of all the spectra taken in that position angle. The values obtained from spectra taken in P.A. $25^{\circ}, 45^{\circ}$, and $175^{\circ}$ are smaller than the others. They come from plate 894 which is less dense than most of the other plates measured.

Aside from the four components described above, other features are present in the [O III] lines but in comparison they are weak and/or indistinct. On deeply exposed spectra, the [O III] lines seem to extend along the continuum on each side of the central wavelength of cloud I. The blue wing is more conspicuous than the red wing and can be seen up to $\sim 15 \AA$ blueward of the wavelength of cloud I. It is likely that these wings originate in the central part of cloud I. They represent gas with motions up to $900 \mathrm{~km} \mathrm{~s}^{-1}$ with respect to the center.

Another weak feature appearing on strongly exposed plates is a straight narrow line seen on $\lambda 5007$ in P.A. $60^{\circ}, 90^{\circ}, 120^{\circ}$ and extending more than $5^{\prime \prime}$ from the continuum source. Its wavelength is difficult to measure; it agrees within $\sim 0.5 \AA$ or $30 \mathrm{~km} \mathrm{~s}^{-1}$ with the central wavelength of cloud I. Ignoring the objection that on our spectra this line does not extend uniformly around the center, we think it likely that it is emitted by the interstellar gas lying in the equatorial plane of the galaxy and which is not directly related to the nuclear clouds, and we assume that this line provides the systemic velocity of NGC 4151. With this assumption, cloud I, which is the brightest component of the cloud complex and which is centered on the continuum source, is at rest with respect to the central region of the galaxy. The discussion of the cloud motion in $\S \mathrm{II} c$ is based on this assumption.

We have observed NGC 1068 with the same equipment as was used for NGC 4151. We found that the $\mathrm{H} \alpha$ line emitted by the interstellar gas in the central region outside the nucleus is easily detectable and consequently more intense than in NGC 4151 . This is consistent with NGC 1068 being of type Sb and NGC 4151 being of type Sa (Humason, Mayall, and Sandage 1956).

\section{c) Geometry and Velocity Field of the Nuclear Cloud Complex}

We emphasize that the distinction into four main clouds is for clarity and does not mean that the clouds are completely separate features.

For the reasons stated in $\S \mathrm{II} b$, it is difficult and sometimes impossible to determine the dimensions of the clouds from the measures on the spectra of the radial extents of the components. Only for cloud III is the dimension certainly larger than the seeing disk. For clouds I and II, the extent of the components may be entirely due to seeing and/or photographic effects. However there is no doubt that the center of cloud II is located $\sim 1 . .5 \mathrm{SW}$ of the continuum source of NGC 4151 in P.A. $240^{\circ}$; cloud IV is seeing limited in size, and is located $\sim 1^{\prime \prime} \mathrm{NE}$ of the continuum source.

Figure 4 shows, in projection on the sky, the location of the four clouds relative to the continuum source apparently situated at the center of cloud I. The outlines of the clouds were obtained by plotting the data of table 2 and drawing smooth curves through the points. To avoid overcrowding the figure, the data points corresponding to component I (which is symmetrical with respect to the center) have not been plotted. Cloud I is represented by a circle of radius 1".6 which is the mean value of the radial 


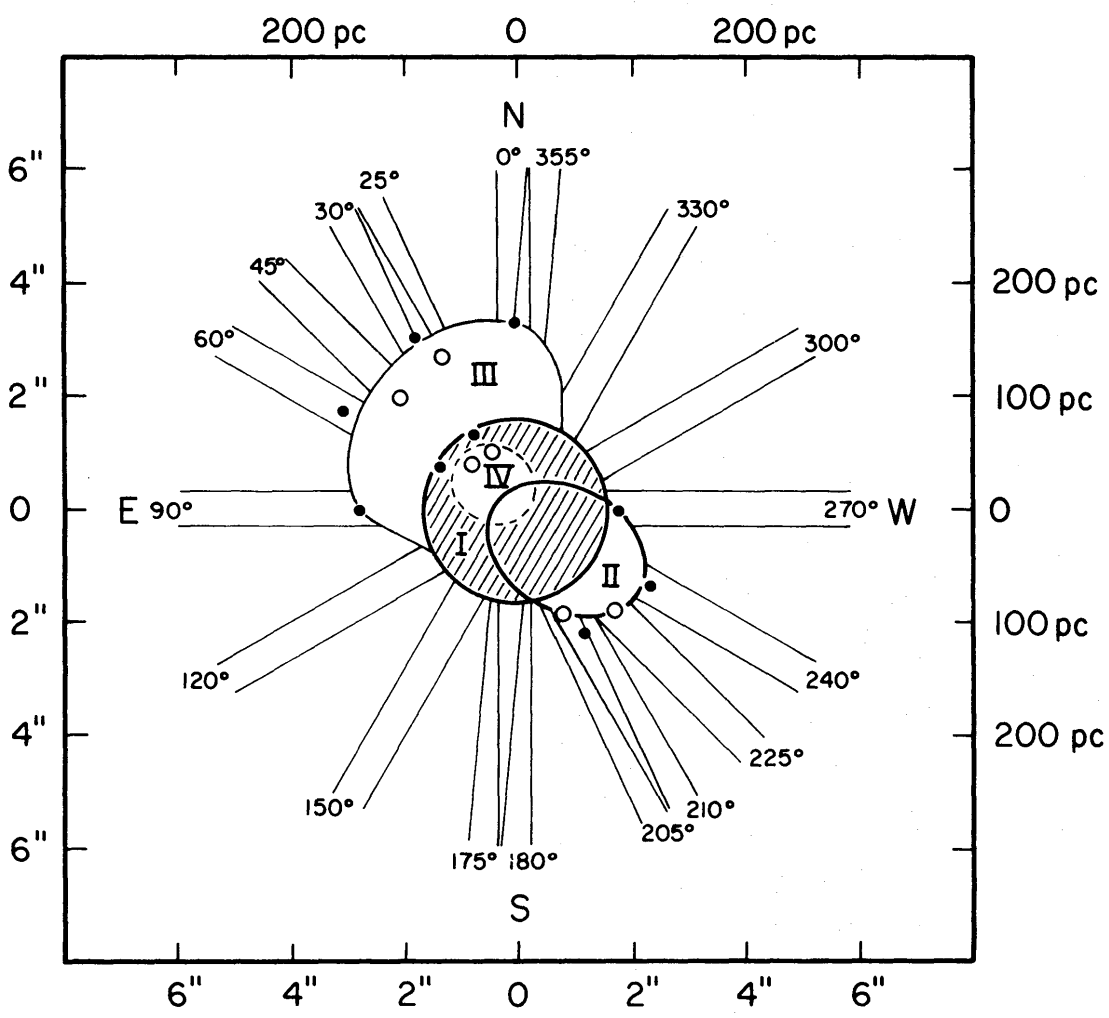

FIG. 4.- The gas clouds in the nucleus of NGC 4151. The contours are deduced from the structure of the [O III] lines on spectra taken in various position angles. Each cloud has the same identification number as the component it emits (see fig. 3). The data of table 2 have been plotted and smooth curves drawn through the points. Open circles, radial extents from plate 894. Black dots, radial extents from the other plates. Data points for cloud I are not shown; this cloud is represented by a circle of radius $1^{\prime \prime} 6$ (see $\S$ II $c$ ). The sets of parallel lines in the position angles in which the spectra were taken represent the projections on the sky of a slit $150 \mu$ wide; this was the widest slit used for the observations. The values of the radial extents expressed in parsecs are based on a value of $10 \mathrm{Mpc}$ for the distance of NGC 4151. We emphasize the fact that the radial extents measured on the plates and listed in table 2 are affected by seeing and/or photographic effects. The radial extents of clouds I and II may be due entirely to these effects; however the center of cloud II is definitely located SW of the center of cloud I at $\simeq 1.5^{\prime \prime}$ from the source of continuum in P.A. $240^{\circ}$. Cloud IV is seeing limited in size and located $\sim 1^{\prime \prime} \mathrm{NE}$ of the continuum source located at the center of cloud I. The apparent major axis of the galaxy lies in P.A. $\simeq 135^{\circ}$.

extent averaged over all position angles. The portion of cloud II which is close to the center has been drawn taking into account that on spectrum 861a component II is slightly west of the center, and on spectrum $861 \mathrm{c}$ it is centered on the continuum. The inner boundary of cloud II is not known.

The value of the central velocity of cloud $I$ is $970 \pm 20 \mathrm{~km} \mathrm{~s}^{-1}$, in good agreement with the value of $980 \pm 20 \mathrm{~km} \mathrm{~s}^{-1}$ determined also from emission lines by Anderson and Kraft (1969).

The differences of radial velocities between the four clouds (see last line of table 2) are interpreted as evidence for expansion motions from the center of NGC 4151: cloud II is moving toward us and cloud III and IV away from us with respect to the center.

The velocity dispersion of the gas inside cloud $\mathrm{I}$, taken to be approximately equal to the half-width of component $\mathrm{I}$, is $150 \mathrm{~km} \mathrm{~s}^{-1}$. On the spectra in the direction of the dispersion, component II overlaps with component I but does not extend beyond the 
central wavelength of component I, as can be seen on spectra taken in P.A. $240^{\circ}$ (see fig. 2a). The velocity inside cloud II ranges from 0 to $360 \mathrm{~km} \mathrm{~s}^{-1}$ with respect to the center of the galaxy, and the apparent radial velocity of the center of cloud II in its outward motion is $180 \mathrm{~km} \mathrm{~s}^{-1}$ with respect to the center of the galaxy. The extended and tenuous cloud III is moving away from the nucleus (and away from us) with an apparent velocity of $180 \mathrm{~km} \mathrm{~s}^{-1}$. In cloud IV the velocity distribution is observed to extend from 150 to $500 \mathrm{~km} \mathrm{~s}^{-1}$ with respect to the center. The small size of this cloud, its apparent proximity to the center, and its relatively large velocity dispersion suggest that it may be younger than clouds II and III.

Cloud II is the brightest after cloud I. A rough estimate of the luminosity of cloud II compared to cloud I can be obtained by using the theoretical value of the intensity ratio [O $\mathrm{III}] \lambda 5007 /[\mathrm{O} \mathrm{III}] \lambda 4959 \simeq 3$. On spectra taken in the NE-SW directions the strength of component II in the line $\lambda 5007$ appears nearly equal to the strength of component $I$ in the line $\lambda 4959$ (see fig. $2 a$ ). Hence cloud $I$ is approximately three times brighter than cloud II, and taking into account some contribution from clouds III and IV, we estimate that within 4 " from the center $\sim 50$ percent of the total luminosity in the [O III] lines originates from the central cloud $\mathrm{I}$.

The distance of NGC 4151 determined from the redshift is $\sim 10 \mathrm{Mpc}$ (taking $H=100 \mathrm{~km} \mathrm{~s}^{-1} \mathrm{Mpc}^{-1}$ ). At this distance $1^{\prime \prime}$ corresponds to $50 \mathrm{pc}$ on the sky.

The distance of the center of cloud II to the continuum source is 1.5 or $75 \mathrm{pc}$ on the sky. By dividing this distance by $180 \mathrm{~km} \mathrm{~s}^{-1}$, which is the velocity of the center of cloud I with respect to the center of the galaxy, one obtains the value of $4 \times 10^{5}$ years which (ignoring projection effect and deceleration) is the time elapsed since cloud II started moving away from the center of the galaxy.

It must be kept in mind that it is not possible to reconstruct the three-dimensional configuration of the cloud complex from only measures of line-of-sight velocities and distances measured in projection on the sky.

The cloud complex has an overall dimension of $6^{\prime \prime}$ or $300 \mathrm{pc}$. It is elongated in a direction close to the apparent minor axis of the galaxy; therefore, the motions of the clouds are not caused by rotation.

\section{DISCUSSION}

Meaningful comparisons can be made between the two Seyfert galaxies NGC 1068 and NGC 4151 because they are both the same distance $(\sim 10 \mathrm{Mpc})$ and have both been studied with similar techniques. The absolute fluxes emitted in the lines [O III] $\lambda \lambda 4959,5007$ are $2.8 \times 10^{41}$ and $2.1 \times 10^{41}$ ergs s$^{-1}$ for NGC 1068 and NGC 4151, respectively (Anderson 1970). The comprehensive study of the velocity field in NGC 1068 made by Walker $(1968 \mathrm{~b})$ has led to the following conclusions concerning the nucleus: three or four clouds are present with internal turbulent velocities with respect to the center of up to $600 \mathrm{~km} \mathrm{~s}^{-1}$. The diameters of these clouds are of the order of 200-350 pc and their characteristic expansion ages of the order of $10^{5}-10^{6}$ years. This, compared with the results presented in $\S$ II $c$, shows that basically the same type of clouds exist in the nuclei of NGC 4151 and NGC 1068 except that the extent of the gas and the velocities are smaller by a factor $\sim 2$ in NGC 4151 .

Anderson and Kraft (1969) found outflowing material moving with velocities 280 , 550 , and $840 \mathrm{~km} \mathrm{~s}^{-1}$ away from the center and toward us, evidenced by the three absorption components they observed in the blue side of the line $\mathrm{He}$ I $\lambda 3889$; our spectra show no features corresponding to this outflowing material. This is consistent with a model in which the absorption is caused by some material which can be denser than in the forbidden-line region and which is lying interior to the emission-line region but external to the continuum source (Cromwell and Weymann 1970; Anderson and Kraft 1971). 
Finally a few comments, qualitative in nature, can be made about the cloud complex in the nucleus of NGC 4151. The four nuclear clouds differ from one another by their velocities with respect to the center, their turbulent velocities, and dimensions; thus it is not possible to know whether the clouds originated in a single or in several explosive events. The nuclear cloud complex has some symmetry with respect to the center: clouds located on opposite sides of the center move in opposite directions. Also, it shows that there is a large-scale inhomogeneity in the distribution of the ionized gas within $150 \mathrm{pc}$ from the center. If the gas is ionized by a central isotropic source, then most of the radiation from this source probably escapes between the clouds. Then, to maintain the clouds ionized, more ionizing radiation has to be emitted by the ionizing source than would be necessary if the gas were concentrated in a single cloud surrounding the source.

I wish to thank Dr. G. Chincarini and Dr. M. Walker for helpful comments on the manuscript. This work was supported by the National Science Foundation through grant GP-25220.

\section{REFERENCES}

Anderson, K. S. 1970, Ap. J., 162, 743.

Anderson, K. S., and Kraft, R. P. 1969, Ap. J., 158, 859.

1971, Ap. J. (Letters), 165, L3.

Cromwell, R., and Weymann, R. 1970, Ap. J. (Letters), 159, L147.

Burbidge, G. R. 1970, Ann. Rev. Astr. and Ap., 8, 369.

Humason, M. L., Mayall, N. U., and Sandage, A. R. 1956, A.J., 61, 97.

Schwarzschild, M. 1971, Bull. A.A.S., 3, No. 2, part 1, 243.

Walker, M. F. $1968 a, A . J ., 73,854$.

1968b, Ap. J., 151, 71 . 